\title{
A simplified two-piece mandibular advancement appliance for obstructive sleep apnea
}

\author{
Hiroshi Ueda ${ }^{1}$, Masato Ueno ${ }^{2}$, Genki Watanabe ${ }^{1}$, Atsushi Horihata ${ }^{1}$, Teruaki Seo ${ }^{2}$, Kazuo Tanne $^{1}$ \\ ${ }^{1}$ Department of Orthodontics and Craniofacial Developmental Biology, Graduate School of Biomedical Sciences, Hiroshima Univer- \\ sity, Hiroshima, Japan; \\ ${ }^{2}$ Chugokushiken Dental Laboratory Co., Hiroshima, Japan. \\ Email: milm@hiroshima-u.ac.jp
}

Received 21 July 2011; revised 23 August 2011; accepted 2 September 2011.

\begin{abstract}
Nowadays mandibular advancement appliances (MAAs) are available and distributed widely in the field of dentistry for the treatment of snoring and obstructtive sleep apnea (OSA). However, a few studies discussed temporomandibular joint (TMJ) discomfort and masticatory muscle stiffness during wearing MAAs are found. A new appliance that we introduced in this pilot study has shown significant jaw movement and could diminish TMJ and masticatory side effects.
\end{abstract}

Keywords: Obstructive Sleep Apnea; Snoring; Mandibular Advancement Appliances; Jaw Movement; Masticatory Muscle Stiffness

\section{INTRODUCTION}

Currently, numerous mandibular advancement appliances (MAAs) are available and distributed widely in the field of dentistry for the treatment of snoring and obstructive sleep apnea (OSA) [1]. It is well known that MAAs are classified roughly into two different types. One is one-piece type and the other is two-piece one. Especially, the latter is widely used to improve temporomandibular joint discomfort and masticatory muscle stiffness during wearing MAAs [2]. However, some conventional two-piece type builds in thick springs or tube-and piston assemblies lingually to the cheeks. OSA patients may have difficulty tolerating this kind of two-piece MAAs.

This article is aimed to describe a simplified and comfortable two-piece MAA for OSA patients.

\section{APPLIANCE DESIGN}

Basically, for the most part, the design of oral appliances for OSA patients is derived from functional orthodontic appliances to enhance mandibular growth in growing patients with small and/or distally-located mandible. This appliance consists of two occlusal splints held together by an orthodontic wire (Figures 1). The splints are cons-

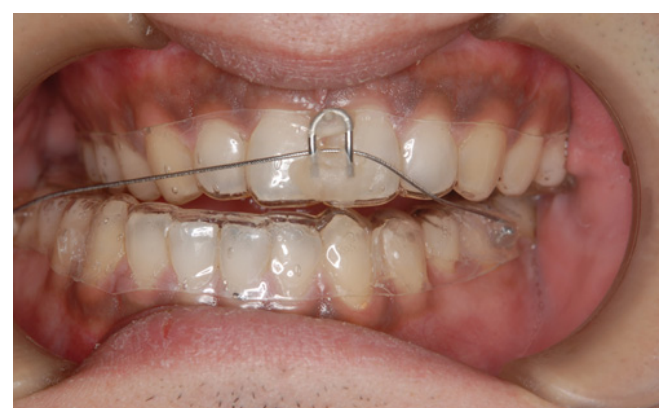

(a)

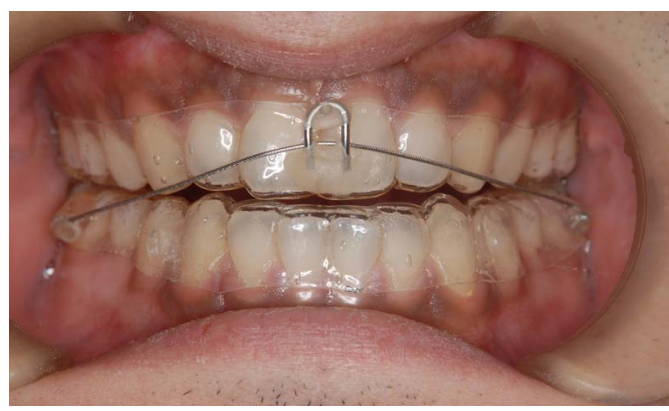

(b)

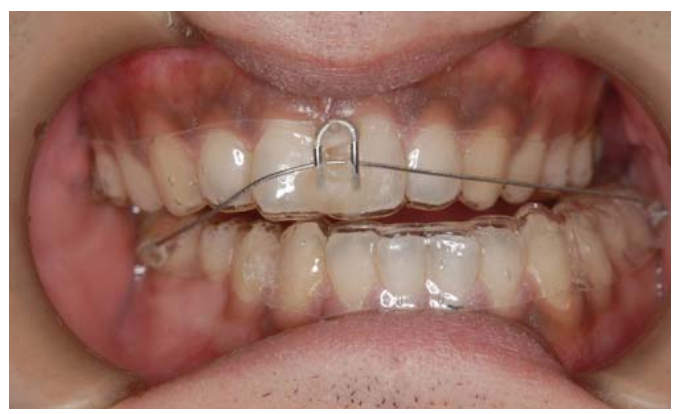

(c)

Figure 1. (a) Mandibular position shifted to the right with MAA; (b) Mandibular rest position with MAA; (c) Mandibular position shifted to the left with MAA. 
tructed of a $0.75 \mathrm{~mm}$ thick acrylic resin that provides full occlusal coverage of teeth. A 0.0175 multi-stranded and twist wire is attached on the buccal sides of the lower splint (Figure 2). Patients can connect the lower splint easily with the hook attached on the front portion of the upper splint. The initial mandibular advancement was defined as two-thirds of maximum mandibular forward position with a $3-4 \mathrm{~mm}$ vertical opening at the anterior teeth. This appliance can permit patients to freely move the jaw horizontally (approximately $10 \mathrm{~mm}$ in the lateral direction) and at the same time prevent the mandible to move downward within $2 \mathrm{~mm}$ in the supine position by means of an optoelectric jaw-tracking system with six degrees of freedom (Gnathohexagraph system II, Onosokki Co., Yokohama, Japan) (Table 1).

The mandibular position can also be titrated forward from its initial position by adjusting the wire length on the appliance.

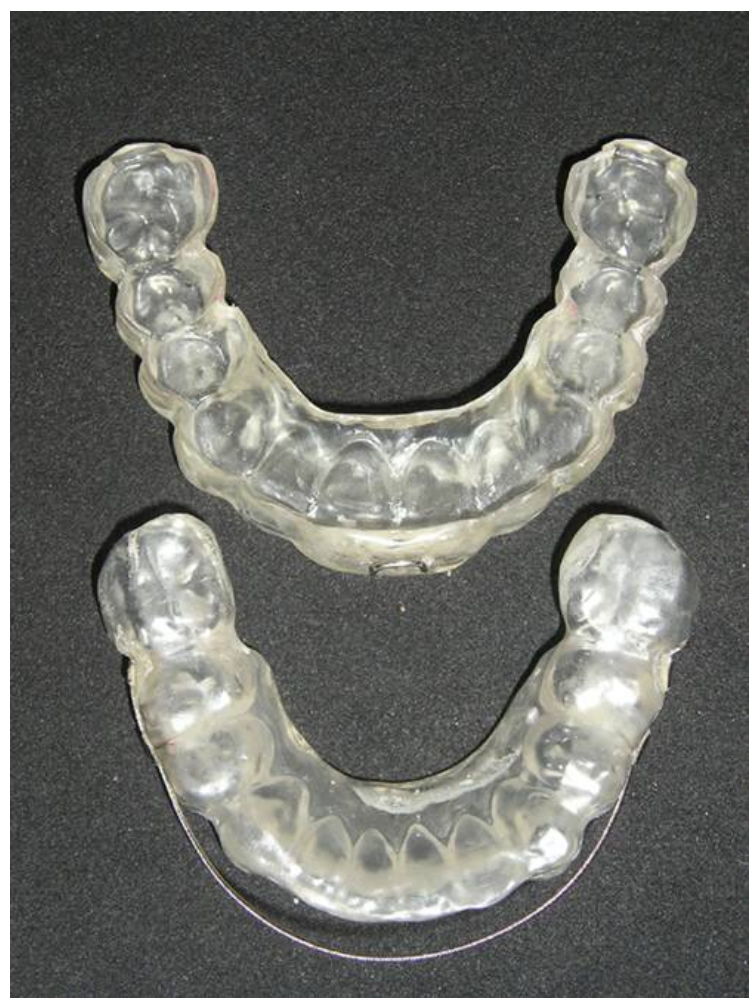

Figure 2. Upper and lower plates of MAA.

Table 1. The distance of mandibular movement with MAA during maximum voluntary effort.

\begin{tabular}{ccc}
\hline & Jaw movement & $\mathrm{mm}$ \\
\hline Right-left direction & Shift to the right & $11.9+/-1.99$ \\
& Shift to the left & $11.4+/-1.82$ \\
Antero-posterior & Maximum jaw opening & $1.63+/-0.95$ \\
direction & Lateral jaw movement & $0.77+/-0.25$ \\
\hline
\end{tabular}

$(\mathrm{N}=5)$

\section{CASE REPORT}

A 38-year-old male with a chief complaint of sleep fragmentation from OSA was referred to our clinic from a cardiac physician. The patient's weight, height and BMI were $73 \mathrm{~kg}, 171 \mathrm{~cm}$ and $25 \mathrm{~kg} / \mathrm{m}^{2}$, respectively.

The molar relationship on both sides was Angle's Class I, and mild crowding was found in the upper and lower anterior teeth (Figure 3). Periodontal problems and temporomandibular joint disorders were not found.

Cephalometric analysis of the patient revealed a skeletal class II tendency (ANB $=5.5$ degree) with large mandibular plane angle (FMA $=42$ degree) (Figure 4).

His apnea-hypopnea index (AHI) was 16.1 (Table 2). Four months after using the two-piece MAA, the patient's initial symptoms were disappeared. Then, the physician repeated PSG examination with the MAA in use. AHI after the use of MAA was 9.2, substantially decreased than 16.1, the initial value. Interestingly, one important result of this case was the MAA therapy exerted a beneficial effect on cardiac rhythm. This patient reported much improvement in the number of events of high and low heart rate according to the heart rate analysis (Table 2). In addition, after the treatment, he has no complaints of temporomandibular joint pain and myofacial discomfort.

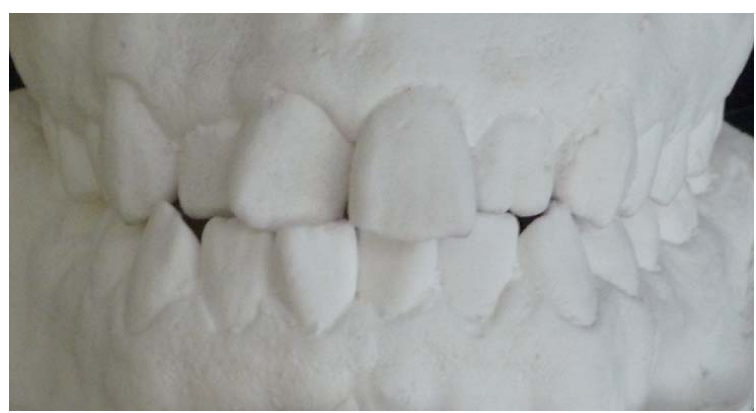

Figure 3. An initial occlusion.

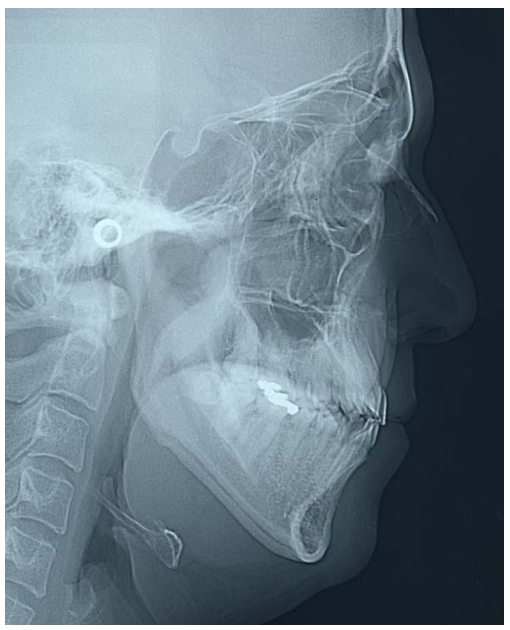

Figure 4. An initial lateral cephalogram. 
Table 2. Polysomnographic results before and after the use of MAA.

\begin{tabular}{ccc}
\hline \multicolumn{2}{c}{ Polysomnographic data } & \\
\hline AHI (/hr) & Before & After \\
\hline Hypopnea (/hr) & 16.1 & 9.2 \\
Obstructive apnea (no.) & 14.5 & 8.7 \\
Central apnea (no.) & 7 & 2 \\
Mixed apnea (no.) & 1 & 0 \\
Minimum SaO 2 & 3 & 1 \\
Snoring (no.) & 82 & 91 \\
Mean Pulse B.P.M. & 835 & 1 \\
Number of events of high heart rate & 67 & 51.4 \\
Number of events of low heart rate & 156 & 8 \\
\hline
\end{tabular}

\section{CONCLUSIONS}

The materials used for the appliance are easy to obtain and the fabrication is extremely simple. This appliance

\section{APPENDIX}

Apnea: cessation of ventilation for 10 seconds or longer.

Hypopnea: decreased airflow for 10 seconds or longer and oxygen desaturation greater than $4 \%$.

AHI (/hr): average number of apneas plus hypopneas per hour of sleep.

Obstructive apnea: absence of airflow despite of persistent breathing efforts. permits to move the mandible greatly in the lateral direction, not so much in the backward direction. Therefore, undesirable side effects such as temporomandibular joint disorders and masticatory dysfunction are less during wearing.

This appliance is efficient, inexpensive, breaking hardly and friendly to intra- and extra-oral tissues. A further investigation focusing on the long-term effect of this appliance in more OSA patients should be attempted.

\section{REFERENCES}

[1] US Food and Drug Administration (1999) Oral devices cleared for treatment of snoring and/or obstructive sleep apneas of 1/7/99. Food and Drug Administration, Washington, DC.

[2] Lowe, A.A. (2000) Dental appliances for the treatment of snoring and/or obstructive sleep apnea. Principles and Practice of Sleep Medicine, 3rd Edition, M. H. Kryger, T. Roth, W. C. Dement, W. B. Saunders Co., Philadelphia.

Central apnea: absence of airflow due to lack of breathing effort.

Mixed apnea: combination of obstructive and central apnea with a central pattern evolving into an obstructive pattern.

Minimum $\mathrm{SaO}_{2}$ : minimum oxygen saturation level.

Mean Pulse B.P.M.: mean heart rate beats per minute. 